\title{
Mechanical Design Considerations for Conventionally Laminated, High-Speed, Interior PM Synchronous Machine Rotors
}

\author{
Edward C. Lovelace, Member, IEEE, Thomas M. Jahns, Fellow, IEEE, Thomas A. Keim, Member, IEEE, and \\ Jeffrey H. Lang, Fellow, IEEE
}

\begin{abstract}
This paper discusses mechanical design considerations that are particular to conventionally (i.e., radially) laminated rotors of interior permanent-magnet synchronous machines. Focus is placed on applications where the radial forces due to high-speed operation are the major mechanically limiting design factor. Proper design of the lamination bridges, or ribs, at the rotor outer diameter is explained in terms of the both material considerations and electromagnetic performance impact. The tradeoff of complexity versus performance associated with using strengthening ribs in the magnet cavities is discussed. The sensitivity of the mechanical design limitations to the rotor-shaft mounting mechanism is also highlighted. These effects are then analyzed using finite-element analysis for a $150-\mathrm{N} \cdot \mathrm{m} / 6-\mathrm{kW}$ integrated starter/alternator designed for operation up to 6000 $\mathbf{r} / \mathbf{m i n}$ with an annular rotor to accommodate a torque converter or clutch assembly. This example demonstrates that it is possible to significantly improve the rotor's structural integrity using the techniques described in this paper with only a very modest impact on the projected machine drive cost.
\end{abstract}

Index Terms-Electrical steel, finite-element analysis (FEA), high speed, interior permanent-magnet (IPM) synchronous machine, laminations, magnetic saturation.

\section{INTRODUCTION}

$\mathbf{R}$ OTOR DESIGN and construction of interior permanent-magnet (IPM) machines is a challenging task due to the conflicting characteristics of improved performance and rotor complexity. IPM machines are of interest because they are particularly attractive from a performance standpoint in traction and spindle applications [1], [2]. IPM machines can be designed with wide, and theoretically infinite, speed ranges for constant power operation with excellent inverter utilization. This is achieved through use of a salient rotor geometry with limited

Paper IPCSD 03-084, presented at the 2001 IEEE International Electric Machines and Drives Conference, Cambridge, MA, June 17-20, and approved for publication in the IEEE TRANSACTIONS ON INDUSTRY APPLICATIONS by the Electric Machines Committee of the IEEE Industry Applications Society. Manuscript submitted for review November 5, 2002 and released for publication January 20, 2004. This work was supported by the MIT Consortium on Advanced Automotive Electrical/Electronic Components and Systems.

E. C. Lovelace is with SatCon Technology Corporation, Cambridge, MA 021421 USA (e-mail: lovelace@alum.mit.edu).

T. M. Jahns is with the Wisconsin Electric Machines and Power Electronics Consortium, Department of Electrical and Computer Engineering, University of Wisconsin, Madison, WI 53706-1691 USA (e-mail: jahns@engr.wisc.edu).

T. A. Keim and J. H. Lang are with the Laboratory for Electromagnetic and Electronic Systems, Department of Electrical Engineering and Computer Science, Massachusetts Institute of Technology, Cambridge, MA 02139 USA (e-mail: tkeim@mit.edu, lang@mit.edu).

Digital Object Identifier 10.1109/TIA.2004.827440 flux contribution from PMs buried within the rotor structure. To achieve the desired degree of saliency, special lamination design and assembly strategies are typically required compared to those required for competing machine types such as surface PM and induction machines.

The rotor design strategies for IPM machines can generally be divided into axially and radially laminated configurations, each with its own advantages [3], [4]. The axially laminated rotor is constructed using many alternating layers of soft and hard magnetic sheets that are laid along the axis of the machine, each bent and individually sized to form the poles of the rotor [1]. This design approach can achieve high-inductance saliency ratios $\left(L_{q} / L_{d}\right)$ in excess of 10:1. However, the axially laminated rotor is relatively expensive to manufacture due to the sorted cutting, shaping, and assembly of the many different laminations that must be employed. Furthermore, a constraining rotor sleeve may be necessary for high-speed operation to prevent lamination intrusions into the air gap. Such sleeves typically reduce the saliency due to their finite thicknesses and often increase losses due to eddy currents when high-strength stainless steel (e.g., Inconel) is chosen for the sleeve material.

By contrast, radially laminated rotors are typically designed with 1-4 layers of hard magnetic material in each pole. Each lamination, as with other conventional machine types, is punched or cut as a single unitary piece for the cross section of the rotor. Cavities are punched or cut into the rotor laminations, and the magnet material is inserted into these cavities. The laminations can be stacked using conventional means so that the rotor is generally easier to manufacture than its axially laminated IPM counterpart.

However, adoption of the radially laminated rotor comes at the expense of saliency with typical inductance ratios ranging from 1.5 up to 10:1, depending on the number of magnet cavity layers and their configuration. For good electromagnetic performance, it is necessary to minimize the steel bridges surrounding the magnetic cavities that are necessary to link the rotor iron segments into a unitary lamination. Each bridge effectively creates a magnetic short ciruit across the PMs, thereby reducing the magnets' contribution to the overall air-gap flux.

This paper examines the mechanical design issues of conventionally (also referred to as transverse or radially) laminated IPM rotors. Only the centrifugal force is considered as this is likely to be the dominant source of mechanical stress in highspeed designs. Each of several key rotor design features are examined in turn with respect to their influence on the rotor stress 


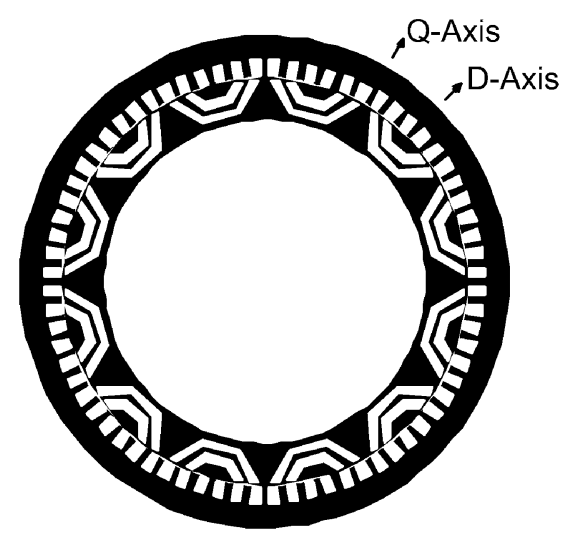

Fig. 1. Cross section of a 12-pole IPM machine.

state and electromagnetic performance. Design strategies with respect to features that can mitigate the resultant mechanical stress state are also presented. The discussion is substantiated through finite-element analysis (FEA) to verify the arguments.

An IPM rotor design for an integrated starter/generator (ISG) application is used throughout the paper to illustrate the significance of these mechanical issues [5]-[7]. A cross section for a 12-pole two-layer design is shown in Fig. 1. In particular, the mechanical stress state of this rotor is a limiting design constraint due to the high rotor tip speed operation that is required of annular direct-drive automotive machinery.

The pertinent design specifications for this ISG design are:

- 6000-r/min maximum operating speed;

- 10000-r/min design burst speed;

- minimum rotor inner diameter (ID) $=165 \mathrm{~mm}$;

- maximum stator outer diameter $(\mathrm{OD})=300 \mathrm{~mm}$;

- bonded PM material in cavities.

\section{Mechanical Design of IPM Rotors}

For the purpose of this discussion, the mechanical design point corresponds to the application specification that produces the worst case mechanical stress in the IPM rotor. The assumptions employed in this development are as follows:

- steady-state speed conditions only;

- temperature effects neglected;

- baseline core material: M19 29-gage electrical steel;

- yield indicated by planar Von Mises stress;

- forces of electromagnetic origin considered negligible;

- vibration and rotor shaft dynamical forces neglected.

With these assumptions, the forces on the rotor are dominated by the steady-state centrifugal forces at constant speed. Therefore, the mechanical design point corresponds to steady-state operation at the design burst speed value, $10 \mathrm{kr} / \mathrm{min}$.

Analytical calculations of the peak stresses due to centrifugal forces acting on a radially laminated IPM machine rotor is a challenging task that is not attempted in this paper due to the complexity of the rotor lamination design features. However, these peak stresses affect the boundaries of the optimization variables that determine the optimal system design, so a qualitative discussion of the resultant forces due to inertial loading is appropriate. The discussion is conducted employing well-

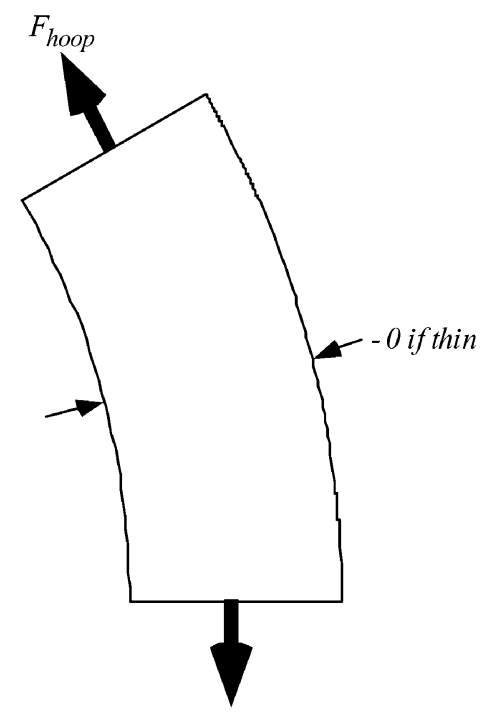

Fig. 2. Sketch of resultant forces on a solid rotor.

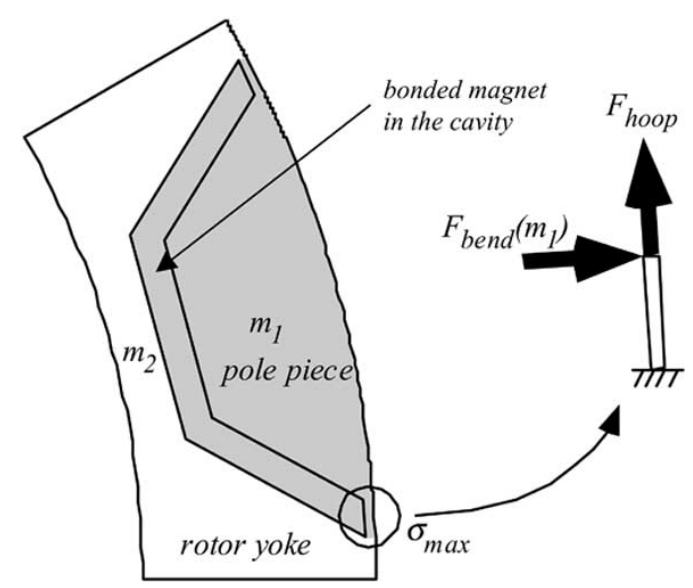

Fig. 3. Sketch of resultant forces on an IPM rotor with one magnet-filled cavity.

known principles that describe the behavior of materials under static loading [8], [9].

Fig. 2 shows a solid rotor cross section with annotations to indicate the major forces on the core due to centrifugal loading. At the simplest level, neglecting the magnet cavities, the rotor resembles a hoop with constant centrifugal loading. Under these conditions, an elemental member of the rotor is under tangential tension and radial compression.

Thin-walled hoop approximations can be justified for modeling the rotor because of the narrow depth of the ISG rotor in comparison to the rotor ID. As a result, the rotor segments mainly experience tangential tension forces. Using this assumption, the major factors affecting the peak stress are the average radius of the "hoop" and the rotational speed. The Von Mises stress increases according to the square of each of these factors.

If the rotor cavities are now considered as in Fig. 3, which only contains one cavity layer, the steel pole piece centered on the $d$ axis is now only attached to the rest of the lamination by the thin steel bridges at each end. Therefore, the centrifugal loading on the pole piece is not evenly distributed around the 




Fig. 4. Sketch of resultant forces on an IPM rotor with multiple layers.

rotor "hoop," causing a substantially radially directed inertial load on the two retaining bridges.

It should be noted that the bonded PM material in the cavity will also contribute to this loading because it is generally less stiff than the steel and will, therefore, contribute additional loading against the inside edge of the pole piece. Therefore, the equivalent magnet mass, $m_{1}$ in Fig. 3 , must be the sum of both the steel pole piece and the magnet (the shaded portion of Fig. 3). The bonded magnet material does not provide any significant bonding between magnet and steel and, therefore, does not transmit force from the yoke to the pole pieces.

The challenge then reduces to modeling the bridges, and this is largely dependent on the specific bridge shape. If the bridges are principally straight, then beam bending approximations are appropriate. When multiple layers are considered as in Fig. 4, each layer can be considered as being independently loaded if the inter-cavity steel sections are wide enough to distribute any stress concentrations between adjacent bridges. The load on each bridge is then the end load in the radial direction due to the inertial loading on the remaining section of the pole piece between the bridge under consideration and the $d$ axis.

If the bridges on each layer have the same dimensions, the bridge at the end of the longest cavity will be under the highest stress. If the cavity ends are rounded as shown in Fig. 5, then the effective length of each "beam" is reduced, and the simple beam approximations described above are no longer reasonable. Each tapered bridge now resembles a round notch stress concentration element under side loading as shown in Fig. 5.

The precise location of the peak stress within each bridge configuration would require significant analysis to determine without resorting to numerical solutions. In particular, the equivalent mounting (fixed or simple) at the ends of each "beam" for the straight-bridge model is not clearly defined. If the ends of each bridge experience minimal bending compared to the rest of the bridge, it is reasonable to assume that the peak stress will be found at the ends. In contrast, the peak stress in the rounded cavity structural model would be expected at the root of the stress concentration, corresponding to the midpoint of each bridge.

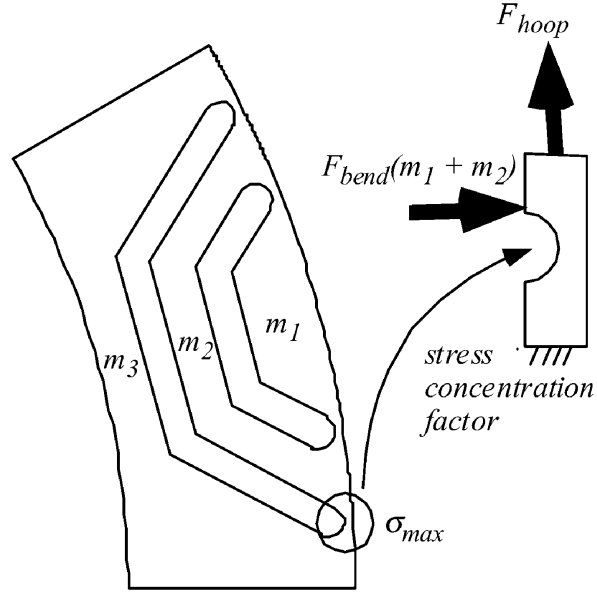

Fig. 5. Sketch of resultant forces on an IPM rotor with multiple cavity layers with rounded tips.

At this stage, some general observations can be made about IPM rotor design decisions that would worsen or improve the mechanical stress conditions.

- Maximum rotor speed-A 10\% reduction in the mechanical design point speed would reduce the peak Von Mises stress by almost $20 \%$.

- Rotor OD-Similarly, a $10 \%$ reduction in the radius at the rotor surface, where the bridges are located, would also reduce the stress by a $20 \%$ factor.

- Rounded bridges-The "beam" stresses are reduced as the "beam" gets shorter with all other dimensions equal. Based on the characteristics of the notch stress concentration model, a circularly rounded bridge shape should nearly minimize the peak stress.

- Smaller pole pieces-A 10\% reduction of the deflecting pole piece mass per unit axial length will reduce the stress almost linearly. This can be achieved by reducing the fraction of the pole pitch that the cavities span. Increasing the number of machine poles can produce the same effect.

- Strengthening rib-Adding a rib redistributes the centrifugal load from the pole piece resulting in a significant improvement in the stress state. A rib that is added to the lamination geometry across the $d$ axis of each cavity resists the centrifugal motion of the pole masses through tension rather than bending.

Another factor in the resultant forces caused by the inertial loading is the effect that the radial deflection of the entire rotor has on the magnitude of the tensile component of hoop stress. The hoop tension in the bridge is due to stretching as the rotor expands into the air gap at higher speeds. The implicit boundary conditions in hoop stress calculations are that the rotor ID and OD boundaries are unconstrained. As a result, reduction of the deflection at either boundary will reduce the expansion of the rotor at the bridge radius and therefore also reduce the hoop stress component of loading.

Constraining the rotor OD is problematic since it would require a material substantially stiffer than steel to decrease the radial deflection under inertial load. Furthermore, adding any 

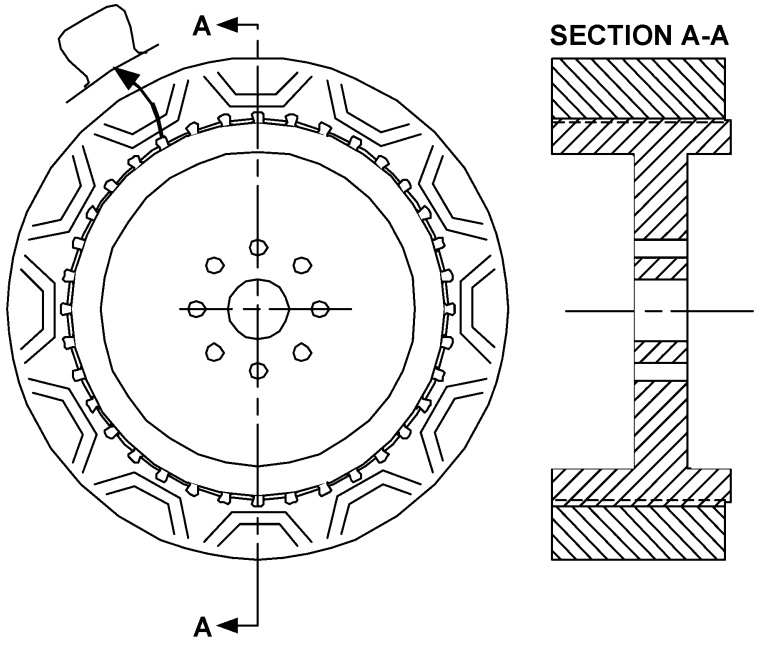

Fig. 6. Rotor hub design using dovetailed joints between the hub and rotor ID.

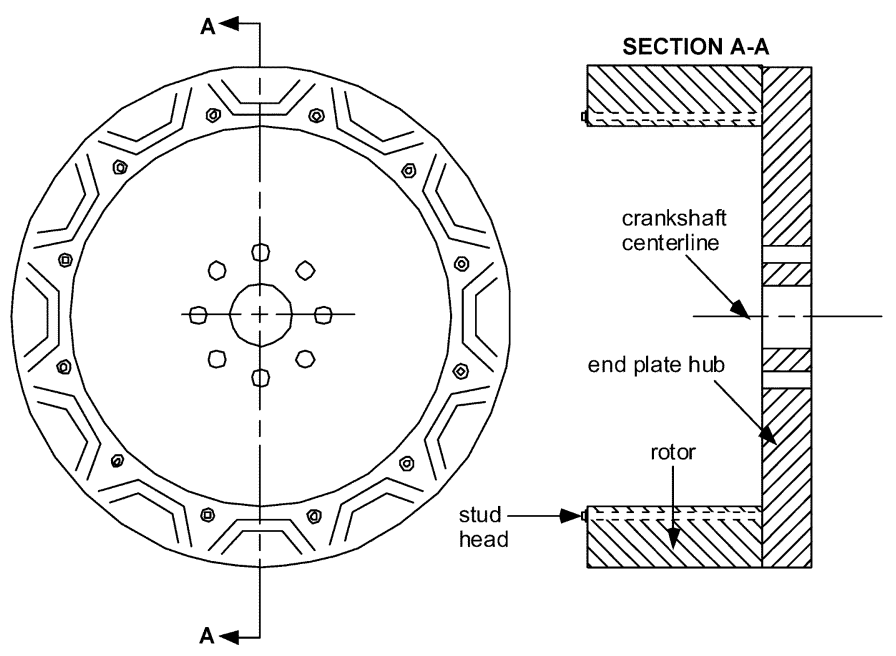

Fig. 7. Rotor hub design using axial bolts through the stack to an end plate.

material in the air gap that adversely affects the electromagnetic saliency of the original rotor would degrade the performance of the machine.

Constraining the rotor ID is a more feasible solution for improving the structural integrity of the rotor. Since there is already a hub that must attach the rotor to the crankshaft, there is an opportunity to specially design the hub to retain the rotor radially. Typically, a hub is only designed to transmit the torque in the circumferential direction as would occur with a hub that is press fit inside the rotor. A press fit, though, does nothing to constrain the rotor ID and so would not mitigate the maximum stress at the mechanical design point.

If there are no space constraints inside the rotor ID, a variety of different hub fixtures might be considered. A welded hub may work but could alter the magnetic properties of the core. One alternative is an axial cylinder that mates with the rotor ID using dovetailed surfaces as shown in Fig. 6. Another alternative is to construct an end plate with studs distributed around the circumference of the end plate (one per pole) as shown in Fig. 7. The laminations would be cut with a hole along each $q$ axis where the core is widest (i.e., there no cavities along the $q$ axis), and then assembled onto the studs.
This bolted system is only practical if sufficient bolt tension can be developed and maintained so that the radial load is taken up by the end plate. If adequate bolt tension is not developed, there will be significant side-loading on the studs that would likely result in shearing off the studs at the surface of the end plate.

The advantage of the dovetail fixture (Fig. 6) or any fixture along the rotor ID surface is that it is structurally robust and nearly symmetric if the radial plate portion of the hub is located axially near the midpoint of the rotor stack. Its chief disadvantage is that the hub cylinder has a finite thickness that may make it necessary to reduce the available space for the rotor laminations.

In contrast, the advantage of an endplate structure (Fig. 7) is that the radial plate is at the end of the stack and does not use any internal real estate inside the ID that might otherwise be reserved for a clutch or torque converter. As a result, this approach may yield the most compact ISG configuration. Furthermore, the absence of the internal hub allows the rotor to be designed with the smallest possible ID and OD, which will reduce the peak stress (squared impact on stress). However, any endplate approach must solve the practical installation problems associated with heavily loaded studs and compressed laminations.

In Section III, the endplate hub structure is analyzed in combination with proposed rotor cross-section modifications to demonstrate a plausible solution for the mechanical design of an IPM machine for the ISG application. The endplate design is chosen for analysis because it allows the smallest machine rotor diameter consistent with the given ISG constraint to provide space inside the rotor ID for a torque converter.

The mechanical design considerations discussed above affect the design performance optimization of IPM machines in several ways [6], [7], [10].

- Rotor diameter-Constraining the rotor diameter and pole piece sizes clearly reduces the available design space for optimization.

- Rotor material-The choice of rotor lamination material affects allowable stress state based on the material yield strength, but it also affects the core losses [11]-[14]. Since the fundamental rotor field is dc, though, the core losses are substantially confined to harmonics introduced by the lamination geometry. The rotor material also influences the required magnet strength for a given PM flux-linkage design because the choice of alloying material content alters the saturation flux density. The saturation flux density, in turn, affects the proportion of the magnet flux that is shorted through the bridges and strengthening ribs.

- Bridge and rib geometry-The geometry of the bridges and strengthening ribs directly affects magnetic performance. For a given IPM machine design, changing from straight to curved cavity tips with the same minimum bridge/rib width increases the flux that is shunted through the bridges and ribs. Adding additional strengthening ribs also increases the proportion of shunted magnet flux. All these bridge and rib factors serve to reduce the available air-gap flux from the permanent magnets, thereby reducing the magnet torque component for a given design or requiring the introduction of larger, stronger magnets. 


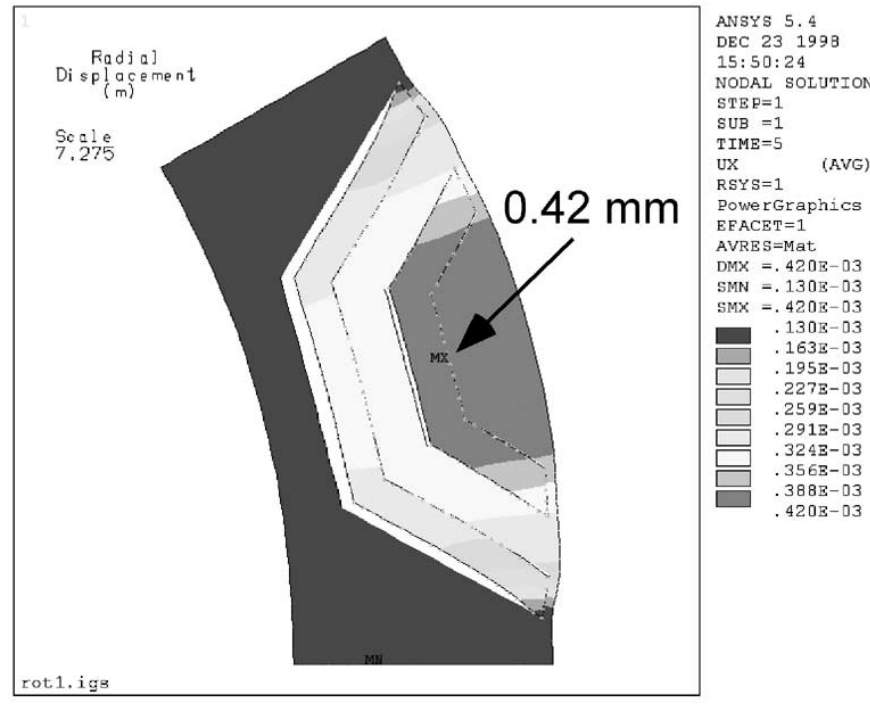

Fig. 8. Radial displacement with straight-edge bridges at $10 \mathrm{kr} / \mathrm{min}$.

For a given design that was initially optimal from an inverter utilization standpoint (i.e., $\lambda_{P M} / L_{d}=I_{a}$ ) [1], design changes subsequently introduced for mechanical reasons shift the machine design point away from the electromagnetic optimum. Ultimately, these changes typically manifest themselves as cost and/or size increases for the machine-inverter combination compared to their comparable values when the machine is optimized for electromagnetic performance alone.

\section{FEA OF AN IPM ROTOR DESIGN}

Structural FEA was conducted to confirm the qualitative understandings presented above [15]. These results are also used as a baseline for scaling to other IPM machine designs provided that the dimensional differences with the baseline are sufficiently small. The FEA was carried out using the ANSYS 2D software package.

Fig. 8 shows the predicted radial displacement of a doublelayer IPM machine at $10 \mathrm{kr} / \mathrm{min}$ without considering any rotor ID deflection constraint due to an attached hub (i.e., a free rotor ID boundary). This cross section is from an ISG with 12 poles and a rotor OD of $246 \mathrm{~mm}$ having straight-edged bridges and no additional strengthening ribs within the two magnet cavities. The plot indicates a maximum deflection on the $d$-axis outer surface of $0.42 \mathrm{~mm}$, which is $60 \%$ of the nominal specified ISG airgap.

The plot confirms that the bulk of the deflection gradients (observed by the change in shading) are in the bridges since the rotor yoke and outer pole piece are both uniformly deflected. This is reasonable because the yoke and pole piece are much thicker than the bridges and so should not bend very much by comparison.

The FEA results show that the bonded magnet material is in nearly continuous contact with the inner boundaries of the two $d$-axis pole pieces. The associated tangential stress plot shown in Fig. 9 predicts a peak stress of $2.6 \mathrm{GPa}$ at the end of the bridge terminating the largest (inner) cavity. Qualitatively, these results match the expected trends quite well. Unfortunately, the

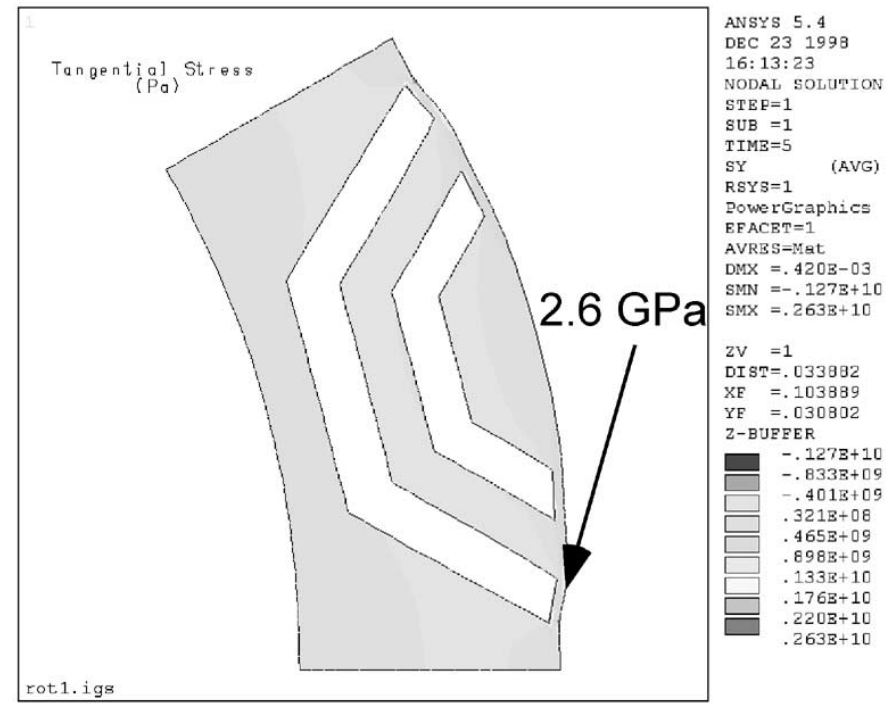

Fig. 9. Tangential stress $[\mathrm{Pa}]$ with straight-edged bridges at $10 \mathrm{kr} / \mathrm{min}$ corresponding to the radial displacement shown in Fig. 8.

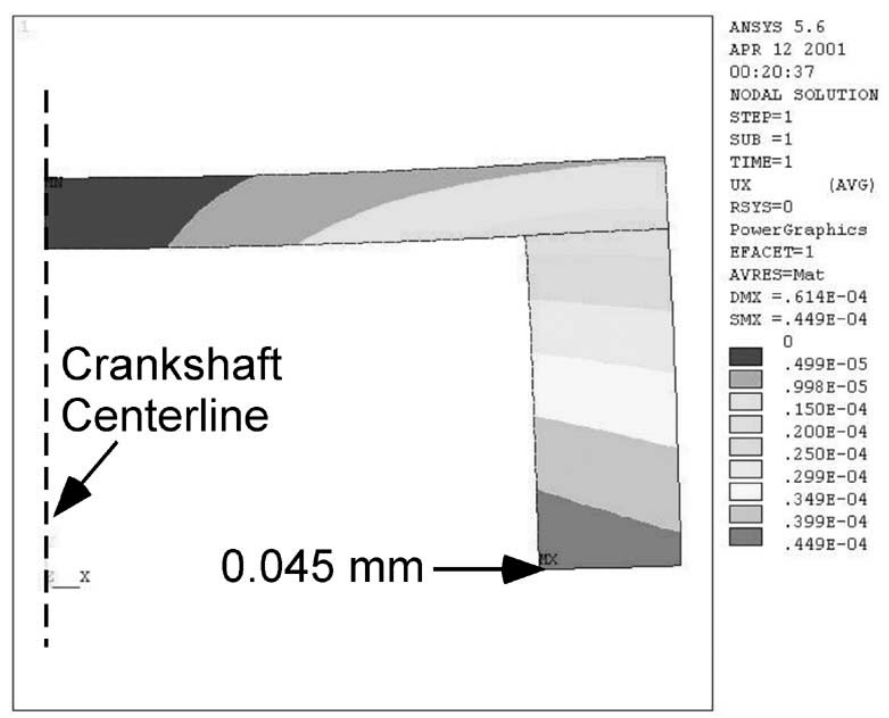

Fig. 10. Predicted deflection of the rotor with endplate hub design in the $R-Z$ plane (axi-symmetric)

peak stress is more than seven times the yield stress of typical electrical steel.

To mitigate the predicted stress, the end plate hub design was analyzed as a means of reducing the radial loading. Furthermore, the machine was re-optimized with the rotor ID fixed to the minimum specified limit $(165 \mathrm{~mm})$, the bridge profiles were rounded, and strengthening ribs were added at the midpoint of each cavity. Fig. 10 shows the deflection of the combined hub-rotor structure in the $R-Z$ plane (with the crankshaft centerline shown on the left. Physically, this FE model is half of the structure shown in Section A-A of Fig. 7. The underlying axi-symmetric model leads to this deflection plot that can be revolved around the crankshaft centerline.

The maximum deflection in the Fig. 10 structure at $10 \mathrm{kr} / \mathrm{min}$ is $0.045 \mathrm{~mm}$ at the far end of the rotor stack from the endplate. This is about $65 \%$ less deflection at the rotor ID than predicted 


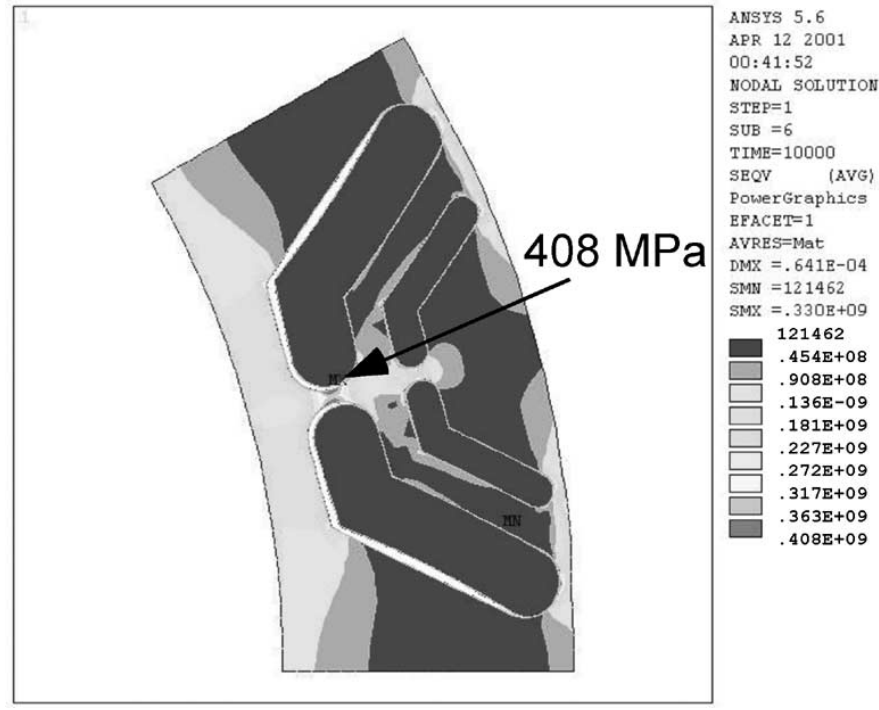

Fig. 11. Von Mises stress at $10 \mathrm{kr} / \mathrm{min}$ with rotor ID deflection constrained by hub.

TABLE I

COMPARISON OF OPTIMIZED IPM FOR AN ISG APPLICATION WITH AND Without MECHANICAL ROTOR CONSIDERATIONS

\begin{tabular}{l|cc}
\hline \multicolumn{1}{c}{} & $\begin{array}{c}\text { Design \#1 } \\
\text { (Fig. 8 and 9) }\end{array}$ & $\begin{array}{c}\text { Design \#2 } \\
\text { (Fig. 10 and 11) }\end{array}$ \\
\hline Bridge Geometry & straight & curved \\
Strengthening Rib & none & centered on d-axis \\
ID deflection & free & limited by hub \\
Stator OD & $300 \mathrm{~mm}$ & $277 \mathrm{~mm}$ \\
Rotor OD & $246 \mathrm{~mm}$ & $216 \mathrm{~mm}$ \\
System Cost & $\$ 589$ & $\$ 604$ \\
Rated Current & $224 \mathrm{Arms}$ & $231 \mathrm{Arms}$ \\
PM Remanence & $0.25 \mathrm{~T}$ & $0.28 \mathrm{~T}$ \\
Saliency Ratio & 5.9 unsaturated & 4.9 unsaturated \\
\hline
\end{tabular}

for the previous configuration in Fig. 8 with an unconstrained rotor ID.

After applying this calculated boundary condition to the rotor ID of an $R-\theta$ plane rotor model, the Von Mises stress was calculated and the results are shown in Fig. 11. This rotor model incorporates the structural design improvements; rounded cavity tips and strengthening ribs at the midpoint of the cavities.

With these design and boundary condition changes, the peak stress at the $10 \mathrm{kr} / \mathrm{min}$ mechanical design point has been reduced by a factor of 6.4 compared to the Fig. 9 design. As a result, the maximum stress on the edge of the rounded bridges and ribs is only 17\% above the yield stress of M19 steel (408 versus $350 \mathrm{MPa}$ ). This may only result in localized plastic yielding because this stress value above yield is not present throughout the width of the bridge and the rib. Though not shown here, analyses were also conducted with a specified deflection of zero on the rotor ID surface, and the resulting Von Mises stress in this case was about $13 \%$ lower than the M19 steel yield strength. Analyzes with no rotor ID deflection constraints developed maximum stress $87 \%$ higher than the yield strength of M19 steel (655 MPa).

The comparison of optimized IPM design for the ISG application both with and without additional mechanical considera- tions for the IPM rotor is shown in Table I. The optimization target employed for this comparison is a system cost model of the machine plus converter that was previously reported on [5], [7], [10]. Design \#1 is the ISG design with the IPM rotor mechanical behavior shown in Figs. 8 and 9, while Design \#2 is the re-optimization with the additional mechanical design constraints represented in Figs. 10 and 11. Table I demonstrates that the ISG design changes for mechanical considerations result in a minor sacrifice for current rating and system cost in exchange for a smaller machine envelope and a machine design that can withstand higher design burst speeds without resorting to more expensive high-strength alloys for the rotor laminations.

All of these results support the conclusion that the IPM machine for the ISG application should be designed with rounded bridge and rib profiles, a minimized diameter, and extra strengthening ribs inside the magnet cavities. Furthermore, the results indicate that additional steps may be required to further reduce the peak stresses within the limits of M19 steel. Re-evaluating the burst speed requirements is another alternative to employing a hub that constrains the rotor ID deflection. A reduction in the mechanical design point speed would provide one effective means of meeting the mechanical strength limit if the automotive application constraints can be modified. For the rotor used in this analysis (Design \#2), the required change in the mechanical design point to meet the M19 yield stress limits without any plastic yielding can be estimated as follows:

$$
10000 \mathrm{r} / \mathrm{min} \sqrt{\frac{350 \mathrm{MPa}}{655 \mathrm{MPa}}}=7310 \mathrm{r} / \mathrm{min} .
$$

This corresponds to $27 \%$ reduction in the maximum speed requirement. With the deflection-constraining hub attachment only a $7 \%$ reduction of the mechanical design speed (9262 r/min) is required to eliminate yielding. Alternatively, different lamination materials with higher yield strengths than M19 steel may also be considered in order to meet the original $10 \mathrm{kr} / \mathrm{min}$ burst speed requirement. Materials of comparable cost with sufficient yield strength, but higher core losses, have been demonstrated for high-speed machinery [14].

\section{CONCLUSION}

This paper has investigated the mechanical behavior and design implications of conventionally laminated IPM rotors with unitary laminations for high-speed applications. Design techniques have been presented that are effective in mitigating the peak rotor mechanical stresses at high speeds. It has been shown that structurally sound designs can be achieved without significant performance degradation through appropriate choices of rotor dimensions, bridge and rib designs, shaft-to-rotor attachment, and materials selection. The example of a high-speed annular ISG application has been used with FEA to substantiate these conclusions. Further work should include prototype testing of structural behavior, evaluation of temperature effects, and structural lumped parameter model development to incorporate directly in the design optimization process. 


\section{REFERENCES}

[1] W. Soong, "Design and modeling of axially-laminated interior permanent magnet motor drives for field-weakening applications," Ph.D. dissertation, Dept. Electron. Elect. Eng., Univ. Glasgow, Glasgow, U.K., 1993.

[2] A. Vagati, A. Fratta, G. Franceschini, and P. M. Rosso, "A.C. Motors for high-performance drives: A design-based comparison," in Conf. Rec. IEEE-IAS Annu. Meeting, 1995, pp. 725-733.

[3] A. Fratta, A. Vagati, and F. Villata, "Design criteria of an IPM machine suitable for field-weakened operation," in Proc. ICEM, 1990, pp. 1059-1065.

[4] T. A. Lipo, T. J. E. Miller, A. Vagati, I. Boldea, L. Malesani, and T. Fukao, "Synchronous reluctance drives tutorial," presented at the IEEE-IAS Annu. Meeting, Denver, CO, Oct. 2-7, 1994.

[5] E. C. Lovelace, T. M. Jahns, J. L. Kirtley Jr, and J. H. Lang, "An interior PM starter/alternator for automotive applications," in Proc. ICEM, vol. 3, Istanbul, Turkey, 1998, pp. 1802-1808.

[6] E. C. Lovelace, T. M. Jahns, and J. H. Lang, "A saturating lumped parameter model for an interior PM synchronous machine," in Proc. IEEE IEMDC, Seattle, WA, 1999, pp. 553-555.

[7] E. C. Lovelace, "Optimization of a magnetically saturable interior permanent-magnet synchronous machine drive," Ph.D., Dept. Elect. Eng. Comput. Sci., Massachusetts Inst. Technol., Cambridge, MA, 2000.

[8] J. E. Shigley and C. R. Mischke, Mechanical Engineering Design, 5th ed. New York: McGraw-Hill, 1989.

[9] L. S. Marks and T. Baumeister, Marks' Standard Handbook for Mechanical Engineers. New York: McGraw-Hill, 1986.

[10] E. C. Lovelace, T. M. Jahns, and J. H. Lang, "Impact of saturation and inverter cost on interior PM synchronous machine drive optimization," in Conf. Rec. IEEE-IAS Annu. Meeting, vol. 1, Phoenix, AZ, 1999, pp. $125-131$.

[11] Nonoriented Sheet Steel for Magnetic Applications, United States Steel, Pittsburgh, PA, 1978.

[12] Alloy Steel Lamination Products, Armco Steel, Pittsburgh, PA, 1999.

[13] Carpenter Alloy Steel Product Selection Guide, Carpenter Steel, Reading, PA, 1997.

[14] W. L. Soong, G. B. Kliman, R. N. Johnson, R. White, and J. Miller, "Novel high speed induction motor for a commercial centrifugal compressor," in Conf. Rec. IEEE-IAS Annu. Meeting, vol. 1, Phoenix, AZ, 1999, pp. 494-501.

[15] ANSYS Multiphysics, ANSYS Corp., Canonsburg, PA, 1999.

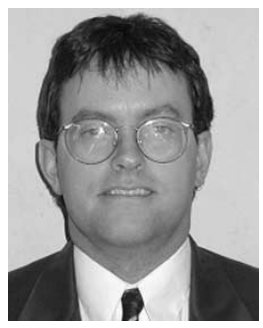

Edward C. Lovelace (S'96-M'00) received the S.B. and S.M. degrees in 1988 and 1996, respectively, from the Department of Mechanical Engineering, and the S.M. and Ph.D. degrees in 1996 and 2000, respectively, from the Department of Electrical Engineering and Computer Science, Massachusetts Institute of Technology (MIT), Cambridge.

In May, 2000, he joined SatCon Technology Corporation, Cambridge, MA, which performs research and development in electric power and energy products, and is currently the Mechanical/Electromagnetics Group Leader. From 1988 to 1996, he was with GE Aircraft Engines, Lynn, MA, where he was involved with development of advanced engine control system designs, military and commercial data communications systems, and ground and flight testing for hydromechanical and digitally controlled engine systems. When he returned to MIT in 1994, he continued to specialize in the design and control of electric machine drives for transportation applications. His current technology research and development interests are mechatronics, advanced machine design, controls, and transportation applications.

Dr. Lovelace received a full three-year fellowship from the Dwight D. Eisenhower Fellowship Program (U.S. Department of Transportation, Federal Highway Administration ISTEA grant and fellowship program) for his doctoral research. Further sponsorship came from the MIT/Industry Automotive Consortium on Advanced Electrical/Electronic Components and Systems with which he was a Research Assistant on automotive electronics research focusing on transition to a $42-\mathrm{Vdc}$ vehicle platform. He was also the recipient of the Technology and Policy Program Cooke Public Service Award and the Best Master Works Oral Thesis Presentation Award.

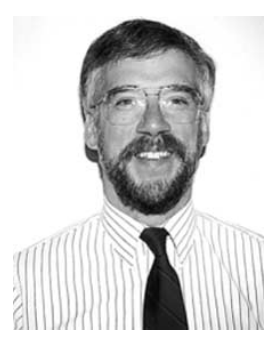

Thomas M. Jahns (S'73-M'79-SM'91-F'93) received the S.B. and S.M. degrees in 1974 and the $\mathrm{Ph} . \mathrm{D}$. degree in 1978 from Massachusetts Institute of Technology, Cambridge, all in electrical engineering.

In 1998, he joined the faculty of the University of Wisconsin, Madison, as a Professor in the Department of Electrical and Computer Engineering, where he is also an Associate Director of the Wisconsin Electric Machines and Power Electronics Consortium (WEMPEC). Prior to joining the University of Wisconsin, he was with GE Corporate Research and Development, Schenectady, NY, for 15 years, where he pursued new power electronics and motor drive technology in a variety of research and management positions. His research interests include permanent-magnet synchronous machines for a variety of applications ranging from high-performance machine tools to low-cost appliance drives. During 1996-1998, he conducted a research sabbatical at Massachusetts Institute of Technology, where he directed research activities in the area of advanced automotive electrical systems and accessories as co-director of an industry-sponsored automotive consortium.

Dr. Jahns was awarded the William E. Newell Award by the IEEE Power Electronics Society (PELS) in 1999. He has been recognized as a Distinguished Lecturer by the IEEE Industry Applications Society (IAS) during 1994-1995 and by PELS during 1998-2000. He has served as President of PELS (1995-1996) and as a Member of the IAS Executive Board between 1992-2001. He was elected Director/Delegate of IEEE Division II, serving during 2002-2003 on the IEEE Board of Directors.

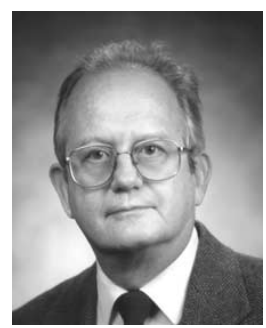

Thomas A. Keim (M'90) received the Doctor of Science degree from Massachusetts Institute of Technology (MIT), Cambridge.

Following graduation, he remained at MIT for several years as a member of the sponsored research staff, working on superconducting electric machines. He then worked for over a decade with General Electric Corporate Research and Development, where he contributed to and led work on superconducting electric machines and on superconducting electromagnets for medical nuclear magnetic resonance imaging. In his next position, he served for nearly a decade at Kaman Electromagnetics as a corporate officer and Chief Engineer. In this position, he led engineering of multiple high-performance electromechanical and power electronics systems, for applications ranging from military propulsion systems and launchers to traction systems for public transit to oil-drilling machinery. Since 1998, he has again been at MIT, where he is a Principal Research Engineer and serves as the Director of the MIT/Industry Consortium on Advanced Automotive Electrical/Electronic Components and Systems. In this capacity, he conducts and directs research into the future of automotive electric power systems. He has authored over 34 publications and is the holder of nine patents.

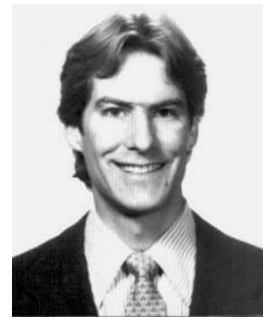

Jeffrey H. Lang (S'78-M'79-SM'95-F'98) received the S.B. and S.M. degrees in electrical engineering and the Ph.D. degree from Massachusetts Institute of Technology (MIT), Cambridge, in 1975, 1977, and 1980, respectively.

$\mathrm{He}$ is a Professor of Electrical Engineering at MIT and Associate Director of the MIT Laboratory for Electromagnetic and Electronic Systems. He has been an MIT faculty member since 1980 . His research and teaching interests focus on the analysis, design, and control of electromechanical systems with an emphasis on rotating machinery, micro sensors and actuators, and flexible structures. He has authored more than 140 technical papers and is the holder of five patents in the areas of electromechanics, power electronics, and applied control.

Prof. Lang has been awarded three Best Paper Prizes by various IEEE Societies. He is a former Hertz Foundation Fellow and a former Associate Editor of Sensors and Actuators. 